\title{
Analysis of the determinants of bba64 (P35) gene expression in Borrelia burgdorferi using a gfp reporter
}

\author{
Aarti Gautam, Marianne Hathaway, Natalie McClain, Geeta Ramesh \\ and Ramesh Ramamoorthy
}

Correspondence

Ramesh Ramamoorthy

rramesh@tulane.edu

Received 13 July 2007

Revised 21 September 2007

Accepted 4 October 2007

\author{
Division of Bacteriology and Parasitology, Tulane National Primate Research Center, Tulane \\ University Health Sciences Center, Covington, LA 70433, USA
}

The bba64 (P35) gene of Borrelia burgdorferi, the agent of Lyme disease, encodes a surface-exposed lipoprotein. The expression of bba64 in vitro is tightly regulated and dependent on several environmental factors. In nature, its expression is induced in the tick vector during feeding and maintained during infection of the vertebrate host. The pattern of expression of bba64 suggests that it imparts a critical function to the pathogen. A previous study has shown that the expression of bba64 is down-regulated in the absence of RpoS, suggesting that the alternative sigma factor may be involved in its expression. A DNA-binding protein has also been shown to specifically recognize a sequence in the 5' regulatory region of the gene. Therefore, the contribution of these putative determinants to the differential expression of bba64 was investigated. The role of RpoS was critically evaluated by genetic complementation of the rpoS mutant using a chromosomally targeted copy of the wild-type gene. The results confirm that RpoS is indeed required for the expression of bba64. The role of the upstream DNA-binding site was examined using bba64 promoter-gfp transcriptional fusions in a shuttle vector. The DNA-binding site was studied by targeting mutations to an inverted repeat sequence (IRS), the most prominent feature within the binding site, as well as by deletion of the entire sequence upstream of the basal promoter. Quantitative assessment of gene expression demonstrated that neither the IRS nor the sequence upstream of the promoter was essential for expression.

Moreover, the expression of the reporter (GFP) appeared to remain RpoS-dependent in all cases, based on the co-expression of GFP and OspC in a subpopulation of spirochaetes and the selective expression of GFP in the stationary phase. Collectively, the data indicate that RpoS is the sole determinant of differential bba64 expression in cultured spirochaetes.

\section{INTRODUCTION}

Borrelia burgdorferi, the spirochaetal agent of Lyme disease, is maintained in nature via a complex enzootic life cycle involving Ixodes ticks and small rodents. To survive in this enzootic cycle, B. burgdorferi must adapt physiologically to diverse environments. Central to its adaptation process is the differential expression of proteins in response to changes in the environment, especially as this organism traverses from its tick vector to the mammalian host and vice versa.

The genome of B. burgdorferi strain B31 is composed of a linear chromosome, nine circular plasmids and 12 linear plasmids (Casjens et al., 2000; Fraser et al., 1997). One of the genetic elements that display prolific differential

Abbreviations: Ab, antibody; IRS, inverted repeat sequence.

A supplementary table listing the reagents and settings used for confocal microscopy is available with the online version of this paper. expression in response to environmental signals is linear plasmid 54 (lp54) (Brooks et al., 2003; Carroll et al., 2000; Clifton et al., 2006; Ojaimi et al., 2003; Revel et al., 2002; Tokarz et al., 2004). lp54 of B. burgdorferi B31 consists of 76 ORFs that include lipoproteins such as OspA and OspB (Barbour \& Garon, 1987) and decorin-binding proteins A (DbpA) and B (DbpB) (Hagman et al., 1998). In addition to these immunogenic proteins, lp54 also carries eight out of the 14 members of gene family 54. Paralogues of this gene family exhibit significant intrafamily sequence divergence, with amino acid similarity and identity values as low as 7.35 and $5.4 \%$, respectively (McDowell et al., 2005). Two members of this family, BBA64 (Gilmore et al., 1997) and BBA66, have been localized to the surface of the spirochaete (Brooks et al., 2006).

Members of gene family 54 display distinct expression patterns. Some members (bba64 and bba66) of the family are silent during the unfed-tick phase (Gilmore et al., 2001; Tokarz et al., 2004) but are turned on during tick feeding 
(Tokarz et al., 2004). Several members (bba64, bba65, bba66, bba73 and bbi36/38) are expressed in the vertebrate host (Gilmore et al., 1997, 2007; Anguita et al., 2000; Liang et al., 2002; Brooks et al., 2006; Clifton et al., 2006; Nowalk et al., 2006). Although the functions of most of the paralogues remain unknown, one member, bba68, is known to bind to human factor H (Kraiczy et al., 2004; Wallich et al., 2005) and impart resistance (Brooks et al., 2005). Recent data indicate that bba68 is not expressed during infection, as inferred from real-time RT-PCR analyses and the absence of an antibody $(\mathrm{Ab})$ response to the protein in infected animals. Moreover, bba68 expression is not dependent on RpoS (McDowell et al., 2006). The differential expression of these genes may be reproduced in culture under conditions that mimic the unfed tick $\left(\mathrm{pH} 8.0,23{ }^{\circ} \mathrm{C}\right)$ or the feeding tick $(\mathrm{pH} 7.0$, $35{ }^{\circ} \mathrm{C}$ ). In general, the expression of bba64, bba65, bba66, $b b a 71$ and $b b a 73$ is upregulated while that of $b b a 69, b b a 70$, bbi36/38 and bbi39/41 is down-regulated under culture conditions that resemble the tick feeding process (Carroll et al., 2000; Clifton et al., 2006; Ojaimi et al., 2003; Ramamoorthy \& Scholl-Meeker, 2001; Revel et al., 2002). The effect of inclusion of blood in the culture medium was largely similar to the effect observed under feeding-tick-like conditions (Tokarz et al., 2004). However, with respect to gene family 54, spirochaetes cultured in implanted dialysis membrane chambers (DMCs) display an expression pattern that resembles neither the flat (unfed) nor the feeding tick (Revel et al., 2002; Brooks et al., 2003).

The regulation of expression of two members of the $g b b 54$ family, bba64 and bba66, has recently been investigated. The expression of bba66 was shown to require the presence of a sequence motif that is the binding site for a sequencespecific DNA-binding protein (Clifton et al., 2006). The expression of bba64 has also been shown to be associated with a sequence-specific DNA-binding activity (Indest \& Philipp, 2000). However, based on their sequence specificities, these two paralogues appear to recruit distinct DNAbinding proteins (Clifton et al., 2006). In the case of bba64, the binding site has been localized to a $43 \mathrm{nt}$ region (designated $k 2$ ) immediately upstream of the -35 element (Indest \& Philipp, 2000). The $k 2$ region harbours two features that may comprise the DNA-binding site, an inverted repeat sequence (IRS) and a downstream poly-T tract. Poly-T tracts have been speculated to be involved in regulating gene expression in B. burgdorferi (Sohaskey et al., 1999; Caimano et al., 2005). In a recent study, the expression of bba64 was found to be down-regulated in an rpoS mutant as compared to its isogenic wild-type parent (Fisher et al., 2005). However, a subsequent study found the expression of bba64 to be uniquely constitutive as compared to other paralogues of this gene family with respect to both culture temperature and culture medium $\mathrm{pH}$ (Clifton et al., 2006). Therefore, the role of RpoS in the expression of bba64 remains somewhat uncertain.

In this study, we critically examined the role of RpoS in the expression of bba64 by complementing the B31 A3rpoS mutant with a wild-type copy of the rpoS gene inserted into the chromosome. We also investigated the role of the upstream sequence, specifically the IRS and the poly-T tract within the $k 2$ region, in the expression of bba64 using $g f p$ as a reporter. The importance of the $k 2$ region in $b b a 64$ expression was examined using a combination of mutations and deletion.

\section{METHODS}

Bacterial strains and culture conditions. Low-passage, infectious B. burgdorferi clones B31 A3 (Elias et al., 2002) and B31 5A4NP1 (cp9 $9^{-}$bbe02: : $\mathrm{kan}^{r}$ ) (Kawabata et al., 2004), as well as the B31 A3 rpoS mutant (Elias et al., 2002) were used in the current study. The Escherichia coli strains Top 10 (Invitrogen) and XL1 Blue MR (Stratagene) were used in the generation of constructs and for the preparation of plasmids for the transformation of B. burgdorferi. E. coli transformants were selected by plating on Luria agar (1.3\%) supplemented with $100 \mu \mathrm{g}$ ampicillin $\mathrm{ml}^{-1}, 10 \mu \mathrm{g}$ gentamicin $\mathrm{ml}^{-1}$ or $100 \mu \mathrm{g}$ spectinomycin $\mathrm{ml}^{-1}$. B. burgdorferi strains and transformants were grown in BSK II $+6 \%$ rabbit serum (Sigma) or in BSK-H complete media (Sigma). Spirochaetes were cultured in $5 \% \mathrm{CO}_{2}, 3 \%$ $\mathrm{O}_{2}$ and $92 \% \mathrm{~N}_{2}$ at $34{ }^{\circ} \mathrm{C}$. The cultures were set up at an initial density of $1 \times 10^{5}$ organisms $\mathrm{ml}^{-1}$ and harvested at stationary phase (1$2 \times 10^{8}$ organisms $\mathrm{ml}^{-1}$ ). Spirochaetal cultures for confocal microscopy were harvested at late exponential phase. Enumeration of cells in culture was performed by dark-field microscopy.

Generation of B31 A3rpos/rpos ${ }^{+}$clones. The rpoS mutant was complemented with a wild-type copy of strain B31 rpos that was targeted to the chromosome at the BB0472-BB0473 intergenic site simply because this presented a large region of sequence with no known function. The first step in the assembly of the complementation construct was the construction of a hybrid bmpA promoter-aadA gene for positive selection of transformants in $B$. burgdorferi. The $b m p A$ promoter $(b m p A p)$ region was amplified with primers T79 and B83 and cloned into pQE30. The aadA coding sequence (conferring streptomycin resistance in B. burgdorferi) (Frank et al., 2003) was amplified from plasmid pAM34 [American Type Culture Collection (ATCC) catalogue no. 77185] using primers T227 and B237, and cloned downstream of the $b m p A p$. The $b m p A p-a a d A$ gene was then transferred to pBR322 by PCR using primers T228 and B237. Next, a $1.3 \mathrm{~kb}$ DNA fragment containing the wild-type rpoS gene and 5' flanking sequence, including the RpoN promoter, was amplified from B31 by PCR using primers T267 and B274. This fragment was cloned downstream of the $b m p A p-a a d A$ sequence. To target the $r p o S$ gene to the BB0472-BB0473 intergenic locus on the chromosome, BB0472 and BB0473 sequences were cloned upstream of $b m p A p-a a d A$ and downstream of $r p o S$, respectively. The primers are all listed in Table 1. The resulting plasmid, designated p472ApSrpoS473, was used to transform the B31 A3rpoS mutant by electroporation, as described elsewhere (Samuels, 1995). After overnight recovery, the electroporated spirochaetes were plated on semisolid BSK-H containing streptomycin $\left(50 \mu \mathrm{g} \mathrm{m} l^{-1}\right)$ and kanamycin $\left(100 \mu \mathrm{g} \mathrm{ml}^{-1}\right)$ (Sung et al., 2000). The plates were incubated at $35^{\circ} \mathrm{C}$ in a candle jar container. Colonies usually appeared 2 weeks after plating. The colonies were transferred to liquid media and subsequently expanded. The integration of the wild-type rpos was confirmed by Western blotting and PCR analysis. Two clones were chosen for further characterization.

bba64 promoter-gfp transcriptional fusion constructs. DNA inserts for cloning were derived in most cases by PCR using 
Table 1. Primers used in this study

\begin{tabular}{|c|c|c|c|c|}
\hline Primer & Sequence $^{*}$ & Location $\dagger$ & Gene(s)/plasmid & Use \\
\hline T79 & agtcgatgactcgagatcgatTAAACAATAGGTTGGTTGATGAAG & -130 to -105 & bтрAр & $b m p A$ promoter \\
\hline T81 & agtcgatgactcgagatcgatACTTCACTTAGACACATTATTTAA & -177 to -153 & $A 64 p$ & pQE30-A64p \\
\hline T88 & CAGCTATGACCATGATTACG & +4246 to +4266 & pBSV & Sequencing \\
\hline T188 & cggggtaccACTTCACTTAGACACATTATTTAA & -177 to -153 & $A 64 p$ & pBSV2G-A64p-gfp \\
\hline T227 & cgtgaagaattcATGAGGGAAGCGGTGATCGT & +1 to +20 & StrR & rpos \\
\hline T265 & CTGTTACAGCCACAGAAAAT & +528 to +548 & $b b 0472$ & rpos \\
\hline T266 & agttcagttgtcgacCTCTGCTTTAATTCGTAGAA & Intergenic region & $b b 0473$ & rpos \\
\hline T267 & gatcagatagctagcGGAGGAAATTGATGGAAACC & -478 to -498 & $b b 0771$ & rpos \\
\hline T239 & cggggtaccTTCTTGTACAATAACAATTTTG & -83 to -61 & $A 64 p$ & pBSV2G-A64pmin-gfp \\
\hline T253 & AGGTTCACCCCTGGAAGAAT & +381 to +362 & $A 64 p$ & RT-PCR \\
\hline T306 & GCATGTATTAACTGTGCTAA & +51 to +70 & $A 64 p$ & RT-PCR \\
\hline B199 & tcgcgatcgGTTCTTTACGATGCCATTGGGATAT & +406 to +382 & pQE30 & pBSV2G-A64p-gfp \\
\hline B237 & actagctagcTTATTTGCCGACTACCTTGGTG & +772 to +792 & StrR & $\operatorname{rpos}$ \\
\hline B272 & gaattcctactcgagATCAGAAATACCTCTCTGCA & Intergenic region & bb0472 & rpos \\
\hline B273 & TTAAGCTCCCСTTTTACACC & +382 to +402 & $b b 0473$ & rpos \\
\hline B274 & tgttgaaccgtcgacAACATGTCATGAATGTCAATG & +798 to +818 & $b b 0771$ & rpos \\
\hline FlaBF & ACAGCTGAAGAGCTTGGAAT & +439 to +458 & $f l a B$ & RT-PCR \\
\hline FlaBR & TTGCTCCAACATGAACTCTT & +538 to +519 & $f l a B$ & RT-PCR \\
\hline
\end{tabular}

* Sequences homologous to genes are in upper-case type and unrelated sequences are in lower-case type. Functional restriction enzyme sites added to the primers are underlined.

$\nmid$ Locations are numbered with respect to the coding sequences of the corresponding genes.

ProofStart enzyme (Qiagen). All inserts were purified from lowtemperature-gelling agarose gels using gel extraction kits (Qiagen). Plasmids for analysis were prepared using Plasmid Miniprep columns (Qiagen) and those used for electroporation were prepared under sterile conditions using Tip100 columns (Qiagen). The bba64 promoter region was amplified by PCR with primers T81 and B87 and cloned into pQE30-gfp (Ramamoorthy et al., 2005) to yield pQE30-A64p-gfp. To generate pQE30-A64p5' $\mathrm{m}$, the bba64 promoter region was amplified in two parts using primer sets T81 and B86 and T82 and B87. The T81/B86 and T82/B87 amplicons were assembled in $\mathrm{pQE30/XhoI/EcoRI} \mathrm{in} \mathrm{a} \mathrm{three-way} \mathrm{ligation} \mathrm{to} \mathrm{yield} \mathrm{pQE30-A64p5'} \mathrm{m}-$ gfp. To generate $\mathrm{pQE30}-\mathrm{A} 64 \mathrm{p5}^{\prime} 3^{\prime} \mathrm{m}$-gfp, the promoter region was amplified from $\mathrm{pQE} 30-\mathrm{A} 64 \mathrm{p} 5^{\prime} \mathrm{m}$ using primers T81 and B121 and cloned into the XhoI/EcoRI sites of pQE30-A64p5'm-gfp.

The shuttle vector derivatives of these constructs were generated as follows. For cloning into pBSV2G (Elias et al., 2003), the fragments (A64p-gfp, A64p5'm-gfp and $A 64 p 5^{\prime} 3^{\prime} m-g f p$ ) were amplified using primers T188 and B199. A minimal promoter construct, A64pmin$g f p$, was generated from pQE30-A64p-gfp using primers T239 and B199. These fragments were all cloned into pBSV2G at the KpnI/PvuI sites, resulting in plasmids pBSV2G-A64p-gfp, pBSV2G-A64p5'mgfp, pBSV2G-A64p5'3'm-gfp and pBSV2G-A64pmin-gfp. Finally, a promoterless $g f p$ construct was also generated for use as a control. The promoter sequences of all constructs were confirmed by sequencing using the T88 primer.

Transformation of $\boldsymbol{B}$. burgdorferi. Plasmid DNAs for electroporation were produced under sterile conditions using Qiagen Tip100 columns. The cells were prepared for electroporation as described elsewhere (Samuels, 1995). Electrocompetent B. burgdorferi was transformed as described elsewhere (Samuels, 1995) with the different promoter- $g f p$ fusion plasmids, with a minor modification. Ten micrograms of DNA was electroporated into $90 \mu \mathrm{l}$ of cells. Immediately following electroporation, the cells were resuspended in $10 \mathrm{ml}$ liquid BSK-H media and incubated overnight at $34{ }^{\circ} \mathrm{C}$ to allow the cells to recover. The transformants were selected according to the limiting-dilution method (Yang et al., 2004). After overnight recovery, the cultures were supplemented with $40 \mathrm{ml}$ fresh BSK-H containing gentamicin $\left(40 \mu \mathrm{g} \mathrm{ml}^{-1}\right)$ and kanamycin $\left(100 \mu \mathrm{g} \mathrm{ml}^{-1}\right)$, and distributed into 96-well tissue-culture plates $(200 \mu$ l per well). Two to three weeks after plating, wells that were positive for dividing spirochaetes were identified by a colour change in the medium, and the presence of viable spirochaetes was verified by dark-field microscopy. The antibiotic-resistant clones were inoculated into $1 \mathrm{ml}$ complete BSK-H medium containing the relevant antibiotics. After 3 days, the transformants were expanded into $15 \mathrm{ml} \mathrm{BSK-H}$ complete media. The $15 \mathrm{ml}$ culture was used for the preparation of freezer stocks and to inoculate fresh cultures for analysis of gene expression.

Generation of rat polyclonal anti-Rpos Ab. To assess RpoS expression, a rat polyclonal anti-RpoS Ab was generated. Briefly, $B$. burgdorferi rpoS was cloned into the pQE30 expression vector (Qiagen) and expressed as a hexahistidine fusion protein in E. coli. Overexpression resulted in an insoluble fusion protein that was purified under denaturing conditions, dialysed to remove urea and then used for the preparation of rat anti-RpoS Ab (Genemed Synthesis). The specificity of the anti-RpoS Ab was verified in E. coli 
using whole-cell lysates prepared from uninduced and IPTG-induced cells carrying the pQE30-his ${ }_{6} r p o S^{B b}$ plasmid. Whereas the Ab showed strong reactivity to a band of $\sim 33 \mathrm{kDa}$ in the induced sample, consistent with the expected size of the fusion protein, there was no reactivity with the uninduced sample (data not shown). The Ab was then titrated to determine the highest dilution of the $\mathrm{Ab}$ that provided the best signal in Western blots (data not shown). A dilution of $1: 200$ provided the best signal.

RNA isolation and RT-PCR. DNA-free RNA was isolated from B31 A3, B31 A3rpoS and B31 A3rpoS/rpoS ${ }^{+}$as previously described (Ramamoorthy et al., 1996). Furthermore, the integrity and concentration of each RNA sample were verified as described previously (Ramamoorthy et al., 1996). About $200 \mathrm{ng}$ total RNA was converted to cDNA in a $10 \mu \mathrm{l}$ volume using Taqman reverse transcription reagents (Applied Biosystems) following the manufacturer's instructions. cDNA synthesis was primed with random hexamers and carried out under the following conditions: $26{ }^{\circ} \mathrm{C}$ for $10 \mathrm{~min}$ followed by $48{ }^{\circ} \mathrm{C}$ for $30 \mathrm{~min}$. The enzyme was inactivated at $95{ }^{\circ} \mathrm{C}$ for 5 min prior to PCR. PCR was performed with $2 \mathrm{ng}$ of each cDNA using ProofStart polymerase (Qiagen) in a volume of $30 \mu \mathrm{l}$. To rule out amplification from DNA, reactions containing RNA without reverse transcriptase were also included with the bba64 primer set. The primers used were as follows: T253 and T306 (bba64), and FlaBF and FlaBR (flaB). The reaction conditions consisted of a $5 \mathrm{~min}, 95{ }^{\circ} \mathrm{C}$ denaturation step, followed by 40 cycles of $95{ }^{\circ} \mathrm{C}$ for $30 \mathrm{~s}, 45{ }^{\circ} \mathrm{C}$ for $30 \mathrm{~s}$, and $72{ }^{\circ} \mathrm{C}$ for $1 \mathrm{~min}$, and then a final extension step at $72{ }^{\circ} \mathrm{C}$ for $10 \mathrm{~min}$.

Western blotting. Whole-cell lysates were prepared from stationaryphase cultures and normalized to an $\mathrm{OD}_{600}$ of 5 , as described previously (Ramamoorthy \& Philipp, 1998). For the analysis of protein expression, $10 \mu$ (unless specified otherwise) of each sample was electrophoresed through a $12.5 \%$ SDS-polyacrylamide gel and the proteins were transferred to nitrocellulose. Following $\mathrm{Ab}$ incubations, protein bands were visualized using the chromogen 4chloro-1-naphthol. The following Abs were used: mAb specific for BBA64 (Indest et al., 1997), rabbit polyclonal anti-GFP Ab (SantaCruz Biotechnology), anti-FlaB mAb H9724 (University of Texas Health Sciences Center, San Antonio), anti-OspC mAb B5 mAb (Mbow et al., 1999) and rat polyclonal anti-RpoS Ab (this study). For quantitative analysis of protein expression, the Western blots were digitized and the intensity of individual bands was quantified by densitometry using Kodak Molecular Imaging Software, version 4.0. All experiments were repeated at least once and the analyses of the pooled data are presented.

Immunofluorescence staining and confocal microscopy. The spirochaetal cultures were spun down and the resulting pellets were washed twice with PBS (Invitrogen) to remove the culture medium. The pellets were resuspended in PBS at a density of $\sim 2 \times 10^{8}$ cells $\mathrm{ml}^{-1}$. A $50 \mu \mathrm{l}$ volume of borrelial suspension containing $\sim 1 \times 10^{7}$ cells was applied to Superfrost Plus slides (Fisher). Smears were airdried, taking care to protect them from exposure to direct light. Slides were fixed in methanol for $10 \mathrm{~min}$. Bacterial smears were blocked for $1 \mathrm{~h}$ in blocking buffer [PBS containing $10 \%$ normal goat serum (Invitrogen), $0.2 \%$ fish skin gelatin (FSG; Sigma) and $0.02 \%$ sodium azide (Sigma)]. The blocking solution was removed by gently flicking the slides before addition of the primary Abs. Primary Abs were diluted to the desired concentration in a PBS-FSG buffer (PBS, $0.2 \%$ FSG, $0.02 \%$ sodium azide) (see Supplementary Table S1). Isotype Ab controls (Dako) in combination with the corresponding secondary$\mathrm{Ab}$-fluorochrome conjugates were also included in the analyses. The slides were washed with PBS buffer after the application of each Ab. All incubations were performed in a dark humidified slide chamber at room temperature. Finally, slides were mounted in anti-quenching medium (Sigma) with premium coverslips (Surgipath) and sealed.
The stained and mounted slides were stored in the dark at $4{ }^{\circ} \mathrm{C}$ until imaging. Imaging was performed using a Leica TCS SP2 true confocal laser-scanning microscope, DMIRE2 (Leica), equipped with three lasers $(\mathrm{Ar}, \mathrm{Ar}-\mathrm{Kr}, \mathrm{He}-\mathrm{Ne})$ that span from the visible to the far-red region of the spectrum. Using Leica software, the fluorescence of individual fluorochromes was captured separately in sequential mode after optimization to reduce bleed through between the channels (photomultiplier tubes). Images of individual channels were also merged to obtain composite images containing all channels.

\section{RESULTS}

\section{The expression of bba64 is dependent on RpoS}

One of our first objectives was to rigorously examine the dependence of bba64 expression on the alternative sigma factor RpoS. Although an earlier study demonstrated that the expression of bba64 was down-regulated in an rpos mutant, that study did not employ complementation to confirm this dependence of gene expression on RpoS (Fisher et al., 2005). Therefore, we set out to complement the rpoS mutation to definitively ascertain the dependence of bba64 expression on RpoS. For complementation, a construct containing a wild-type copy of the rpoS (bb0771) gene inserted into the bb0472-bb0473 intergenic site (Fig. 1) was used to transform B31 A3rpoS (Elias et al., 2002), the same strain used in the earlier study. The complemented clone, B31 A3rpoS/rpos ${ }^{+}$, was characterized by PCR using total DNA. The PCR amplification patterns were consistent with the expected genotype (data not shown). Plasmid profile analysis confirmed the presence of all plasmids that were present in the parental strain, B31A3 (Elias et al., 2002). Moreover, the complemented clones displayed normal growth kinetics in BSK-H and BSK II media.

The expression of RpoS in the complemented strain was examined by Western blotting using a rat monospecific anti-RpoS Ab. Both clones exhibited a band similar in size to the wild-type band and consistent with the expected size of the protein $(31 \mathrm{kDa})$. This band was absent in the rpoS mutant strain (Fig. 2a, RpoS). To further confirm RpoS expression in the complemented clones, we also tested the samples for the presence of OspC, a known RpoSdependent protein (Hübner et al., 2001). As expected, the presence of RpoS in the complemented clones restored the expression of OspC (Fig. 2a, panel OspC). Finally, we examined the samples for the presence of BBA64 using an anti-BBA64 mAb (Gilmore et al., 1997). Whereas no BBA64 expression was detected in the absence of RpoS (Fig. 2a, panel BBA64, lane 2), this protein was clearly present in the two complemented clones at a level similar to the wild-type level (Fig. 2a, panel BBA64, lanes 3 and 4). The dependence of bba64 expression on RpoS was further verified at the mRNA level by RT-PCR. The bba64 sequence could be amplified from RNA derived from the wild-type and the complemented strains, but not from the rpoS mutant (Fig. 2b, panel bba64). In contrast, the constitutively expressed $f l a B$ transcript was present in all samples examined (Fig. 2b, panel flaB). These results 


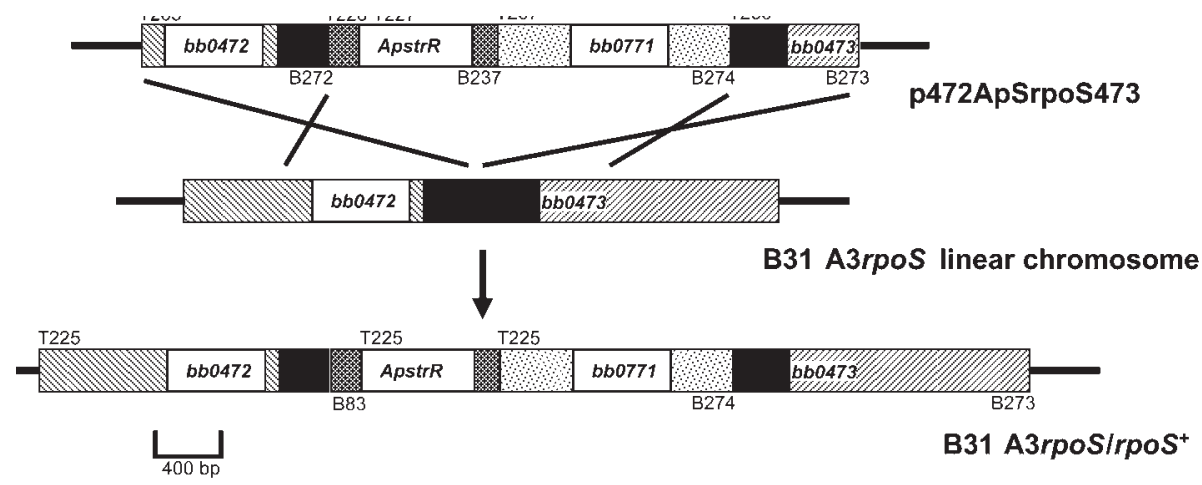

Fig. 1. Strategy for the complementation of the B31 A3 rpoS::Kan mutant. Primers used in the construction of p472ApSrpoS473 are shown. Also shown are the primers used for confirming the genotype of the B31 ${\mathrm{A} 3 r p o S / r p o S^{+}}$strain. For more information on the $\mathrm{T}$ and $\mathrm{B}$ primers shown here, please refer to Table 1 . The bb0472-bb0473 intergenic region, defined as the sequence between the stop codon of $b b 0472$ and the start codon of $b b 0473$, is shown as a filled box. The $b m p A$ promoter-aadA hybrid gene is designated ApstrR in the figure.

conclusively establish the dependence of BBA64 expression on the alternative sigma factor RpoS under conditions of high cell density.

\section{A minimal promoter is sufficient for optimal expression of bba64 in culture}

The presence of any functional sequence elements in the $5^{\prime}$ regulatory sequence of bba64 was assessed by a combination of deletions and mutations. A previous study identified a $43 \mathrm{nt}$ region (designated $k 2$ ) immediately upstream of the -35 element as the binding site for a bba64-specific DNA-binding activity (Indest \& Philipp, 2000). This region is characterized by an IRS terminating in a poly-T tract. Presumably, the IRS is the site of interaction with the DNA-binding protein. Therefore, the IRSs were mutated, either singly $\left(A 64 p 5^{\prime} m-g f p\right)$ or in combination (A64p5' $\left.3^{\prime} m-g f p\right)$ (Fig. 3). To further assess the importance of the $k 2$ region and any other potential regulatory sequences upstream of the promoter, another construct was generated in which the sequence upstream of the -35 was entirely deleted (A64pmin-gfp) (Fig. 3). The expression of the marker $g f p$ gene from these constructs was compared to the expression of $g f p$ from a wild-type construct (A64p$g f p$ ) and a promoterless construct ( $g f p$ ) (Fig. 3). For the assay of gene expression, the promoter- $g f p$ fusion constructs were assembled in the E. coli-B. burgdorferi shuttle vector pBSV2G. The shuttle vector constructs were introduced into B. burgdorferi $\mathrm{B} 31$ 5A4NP1, a highly transformable and infectious strain (Kawabata et al., 2004). Moreover, the presence of a kanamycin-resistance determinant on linear plasmid 25 (lp25) provides positive selection for the presence of this plasmid in transformants. Determinants on the lp25 plasmid have been shown to be essential for virulence (Grimm et al., 2004; Labandeira-Rey \& Skare, 2001; Purser \& Norris, 2000). Therefore, all transformants were selected with kanamycin to ensure the presence of lp25.

For each construct, two transformants were examined. The expression of GFP was estimated by quantitative Western blotting. The samples were probed with Abs specific for GFP, BBA64, FlaB and RpoS. The GFP bands in individual samples were quantified by densitometry (Fig. 4a, panel GFP) and normalized to the corresponding FlaB bands (Fig. 4a, panel FlaB). The normalized values were then (a)

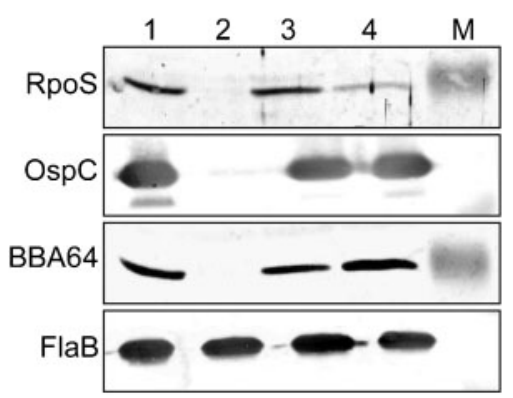

(b)
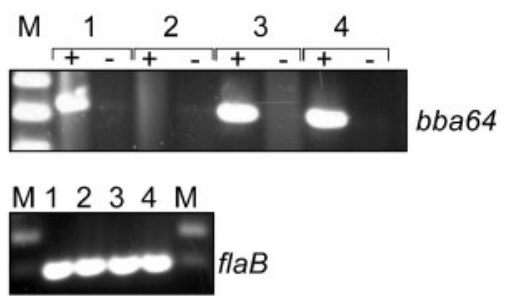

Fig. 2. BBA64 expression is dependent on RpoS. (a) Expression of the RpoS, BBA64, $\mathrm{FlaB}$ and OspC proteins was examined by Western blotting. (b) Expression of bba64 and flaB mRNAs was examined by RT-PCR. In the case of bba64, the RT-PCRs for the test samples (+ lanes) as well as negative control reactions in which the reverse transcriptase was omitted (- lanes) are shown. Lanes: 1, B31 A3 wild-type; 2, B31 A3rpoS; 3 and 4, B31 A3rpoS/rpoS ${ }^{+}$clones 1 and 2, respectively. $M$, protein (a) and DNA (b) markers. 
(a)

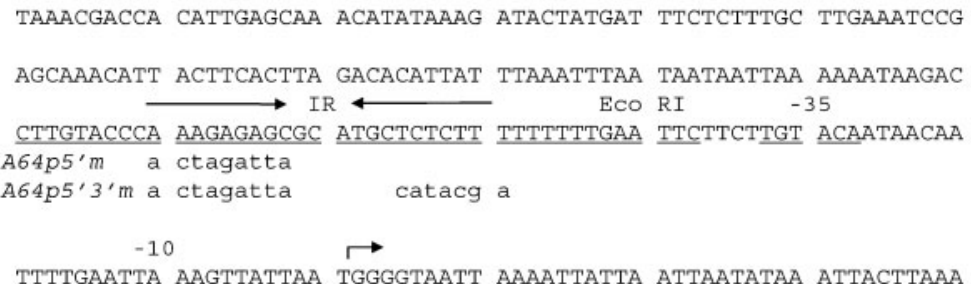
ATTTTTATTG

(b)

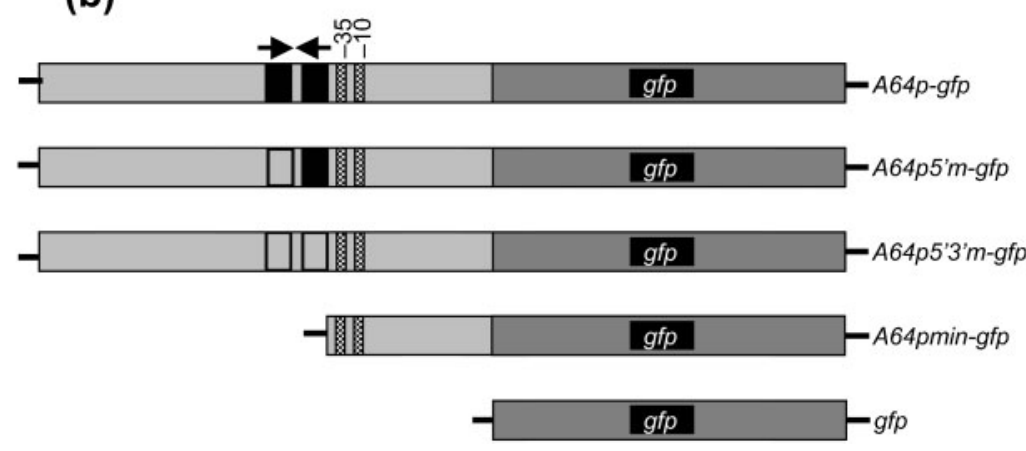

Fig. 3. (a) Sequence and features of the bba64 upstream region. The transcription start site (bent arrow) (Indest et al., 1997), the associated -10 and -35 elements (underlined), and the translation start codon (indicated in bold type) are shown. The IRSs are indicated by arrows above the $k 2$ DNA-binding region (underlined). The mutations in the two promoter variants $A 64 p 5^{\prime} \mathrm{m}$ and $A 64 p 5^{\prime} 3^{\prime} \mathrm{m}$ are shown below the corresponding wild-type sequence. The A64pmin promoter variant contains the sequence downstream of the EcoRl site (GAATTC) and includes the EcoRI site. (b) A diagrammatic representation of the different promoter constructs used in the study. The wild-type IRSs are indicated by filled boxes, whereas the open boxes indicate that these sites have been mutated.

expressed relative to the wild-type promoter construct $(A 64 p-g f p)$ (Fig. 4b). As expected, no GFP expression was detectable in the absence of the bba64 promoter (Fig. 4a, panel GFP, lane 1). In contrast, the GFP band was evident for all of the promoter constructs (lanes 2-4). However, surprisingly, the expression of GFP from the two mutant constructs $A 64 p 5^{\prime} m-g f p$ and $A 64 p 5^{\prime} 3^{\prime} m-g f p$, as well as from the minimal promoter construct A64pmin-gfp, was similar to the level of expression derived from the wildtype promoter construct $(A 64 p-g f p)$ (Fig. 4b). To further ensure that the levels reflected the true transcription potential of these fusions and were not the consequence of other determinants, these samples were also screened with Abs specific for BBA64 and RpoS. All samples were positive for both proteins, and more importantly, with the exception of the wild-type construct, which exhibited slightly lower levels (75\% of that of the other samples) of both RpoS and BBA64, the levels of these two proteins were similar in all other samples, including the promoterless $g f p$ fusion (Fig. 4a, panels RpoS and BBA64).

\section{GFP from all promoter constructs is co-expressed with OspC in a subpopulation of spirochaetes}

We showed that the expression of the bba64 gene is RpoS dependent and that the expression of the endogenous rpoS and bba64 genes in the transformants harbouring the various fusion constructs is similar. These results are consistent with RpoS also being involved in the expression of GFP from the fusion constructs. To further examine the nature of $g f p$ expression from the various pBSV2G bba64 promoter constructs, we resorted to confocal microscopy. (a)

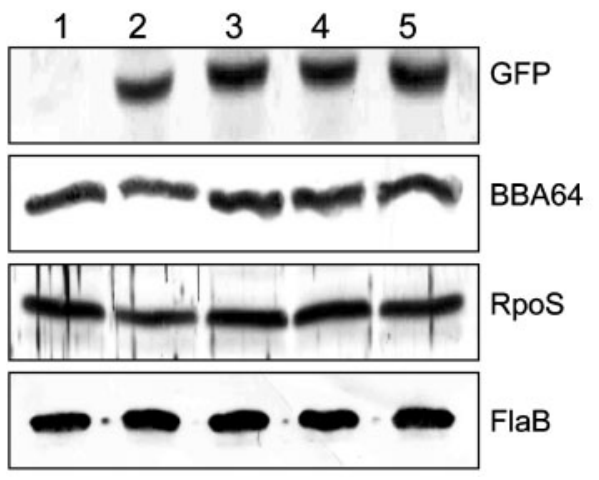

(b)

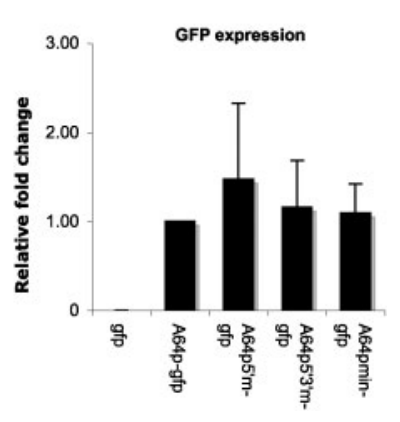

Fig. 4. Influence of the bba64 upstream elements on GFP expression. (a) Expression of FlaB, GFP, BBA64 and RpoS was detected by Western blotting using specific Abs. For the detection of GFP, BBA64 and RpoS, sixfold greater volumes were loaded than the volume of sample used for the detection of FlaB. Lanes: 1, pBSV2G-gfp; 2, pBSV2G-A64pgfp; 3, pBSV2G-A64p5'm-gfp; 4, pBSV2GA64p5'3'm-gfp; 5, pBSV2G-A64pmin-gfp. (b) Relative expression of GFP from the different constructs. The SEM for each sample is indicated by the error bars. 
The rationale for using confocal microscopy was based on the following observations. First, the expression of both ospC and bba64 is dependent on RpoS (Hübner et al., 2001; Fisher et al., 2005; Yang et al., 2005; this study). Second, only a proportion of cultured spirochaetes stain positive for expression from an ospC promoter (Carroll et al., 2003) or OspC (our unpublished observations). We speculated that the expression of RpoS in cultured spirochaetes, for unknown reasons, is limited to a subpopulation, and consequently results in the selective expression of OspC. We therefore examined the populations of transformed spirochaetes for the expression of GFP, OspC and BBA64 proteins by confocal microscopy.

We first examined the relationship between BBA64, GFP and OspC expression at the population level using pBSV2G-A64p-gfp-transformed B31 5A4NP1 spirochaetes. Slides containing these spirochaetes were stained with an
anti-OspC mAb (Mbow et al., 1999) followed by a rabbit polyclonal anti-B. burgdorferi $\mathrm{Ab}$, and subjected to confocal microscopy. The Abs, dilutions and wavelengths used are listed in Supplementary Table S1. The expression of both GFP and OspC was found to be limited to a subpopulation of cells. The green fluorescence of GFP was noticeable in only some spirochaetes (Fig. 5, compare panel GFP and panel $\mathrm{Bb}$ ) against a teeming background of spirochaetes that appeared negative for GFP [panel $\mathrm{Bb}+\mathrm{GFP}$; the overlap of GFP (green) and Bb (blue) appears as sea green]. Similarly, the expression of OspC was also restricted [panels OspC (red) versus $\mathrm{Bb}$ (blue), and $\mathrm{Bb}+\mathrm{OspC}$; overlap appears pink]. Most notably, cells with the OspC $\mathrm{C}^{+}$ phenotype congregated with cells that exhibited a $\mathrm{GFP}^{+}$ phenotype (panel $\mathrm{Bb}+\mathrm{GFP}+\mathrm{OspC}$; the overlap of the three colours appears as yellow staining). In the second experiment, we examined the relationship between OspC
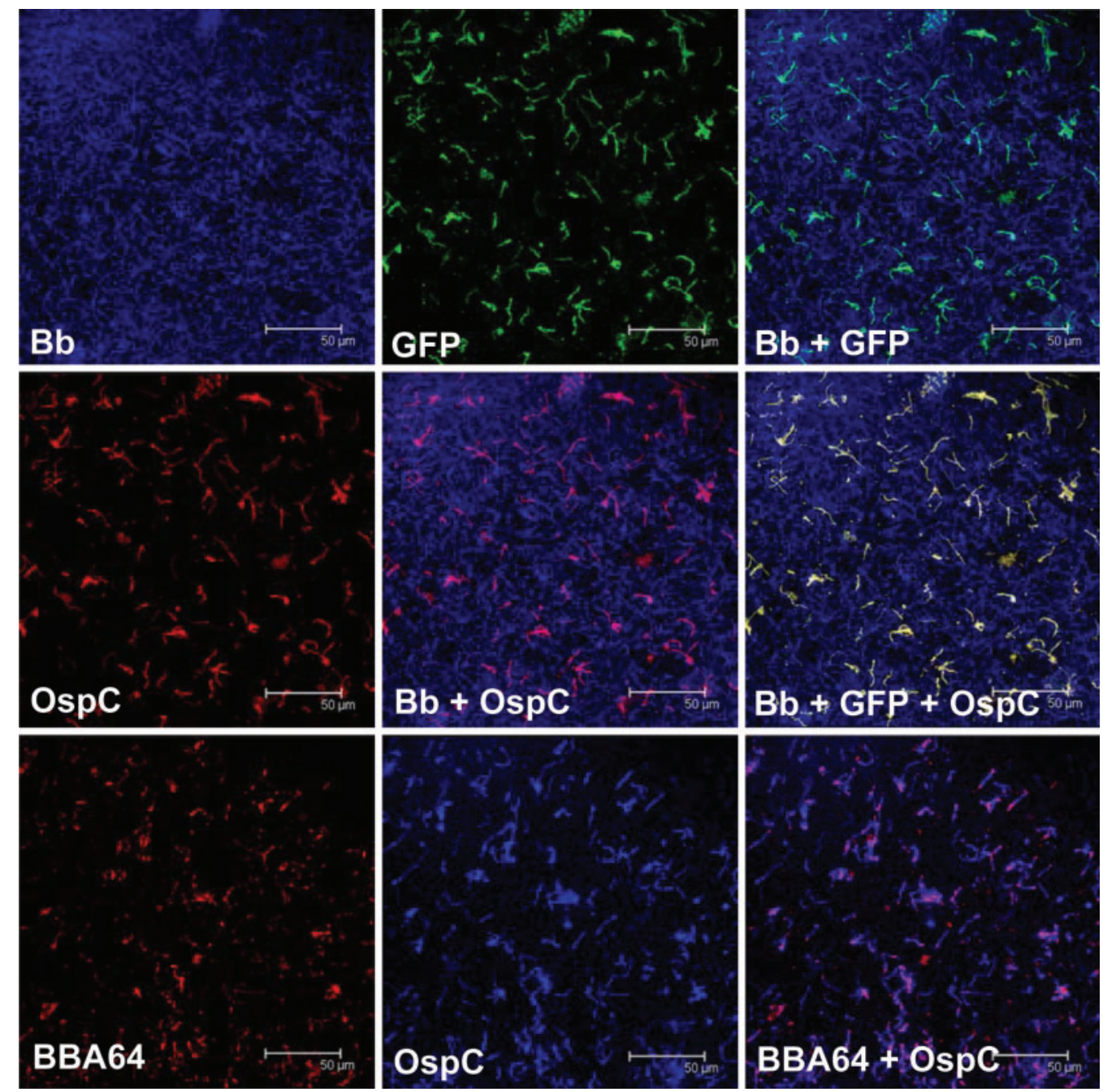

Fig. 5. Confocal microscopic imaging of spirochaetal populations expressing GFP, OspC and BBA64 proteins. Slides containing B31 5A4NP1/pBSV2G-A64p-gfp spirochaetes were stained with Abs specific for OspC (red) and B. burgdorferi (blue), or with Abs specific for BBA64 (red) and OspC (blue), and analysed by confocal microscopy. The individual images were merged to obtain composite images to visualize the co-expression of the proteins in individual cells. 
and BBA64 in spirochaetal populations. Slides were stained first with the mouse anti-BBA64 Ab followed by the antiOspC Ab. Again, only a limited number of spirochaetes appeared positive for BBA64 (panel BBA64, red) or OspC (panel OspC, blue), but more importantly, these two subpopulations were the same (panel BBA64+OspC; the overlap appears as pink staining). Taken together, these results indicate that the same subpopulation of spirochaetes express all three proteins, GFP, BBA64 and OspC.

We next analysed spirochaetes harbouring the other promoter constructs to determine if the co-expression of GFP and OspC seen in B31 5A4NP1/A64p-gfp spirochaetes extended to the other spirochaetes as well. Slides were prepared from each of the five transformed clonal populations: promoterless $g f p, A 64 p-g f p, A 64 p 5^{\prime} m-g f p$, $A 64 p 5^{\prime} 3^{\prime} m-g f p$ and A64pmin-gfp, and stained for OspC. As expected, GFP fluorescence was not detected in spirochaetes harbouring the promoterless $g f p$ construct (Fig. 6, column 1 GFP or 1GFP + OspC). In contrast, GFP fluorescence was clearly visible in numerous spirochaetes transformed with all of the other bba64 promoter constructs (Fig. 6, columns 2-5). Most notably, in all four cases, the same subpopulations stained positive for both
GFP and OspC (panels GFP + OspC). These results suggest that the expression of GFP and OspC shares a common feature that is maintained in all the GFP-expressing clones analysed in this study.

\section{DISCUSSION}

In this study, we characterized the expression of bba64, one of the well-known members of a family of genes that exhibit prolific differential expression in culture under different conditions that are meant to simulate the natural history of this organism. We examined the requirement for RpoS as well as that for an upstream region previously determined to be the site of binding of a bba64-specific DNA-binding protein. The requirement for RpoS was critically evaluated by complementing the B31 A3rpoS mutant with a wild-type copy of the gene targeted to the chromosome. Our study complements a recent comparison of global gene expression in strain B31 A3 and its isogenic rpoS mutant in which it was demonstrated that bba64 gene expression is down-regulated in the rpoS mutant relative to the wild-type (Fisher et al., 2005). A subsequent report found bba64 to be constitutively
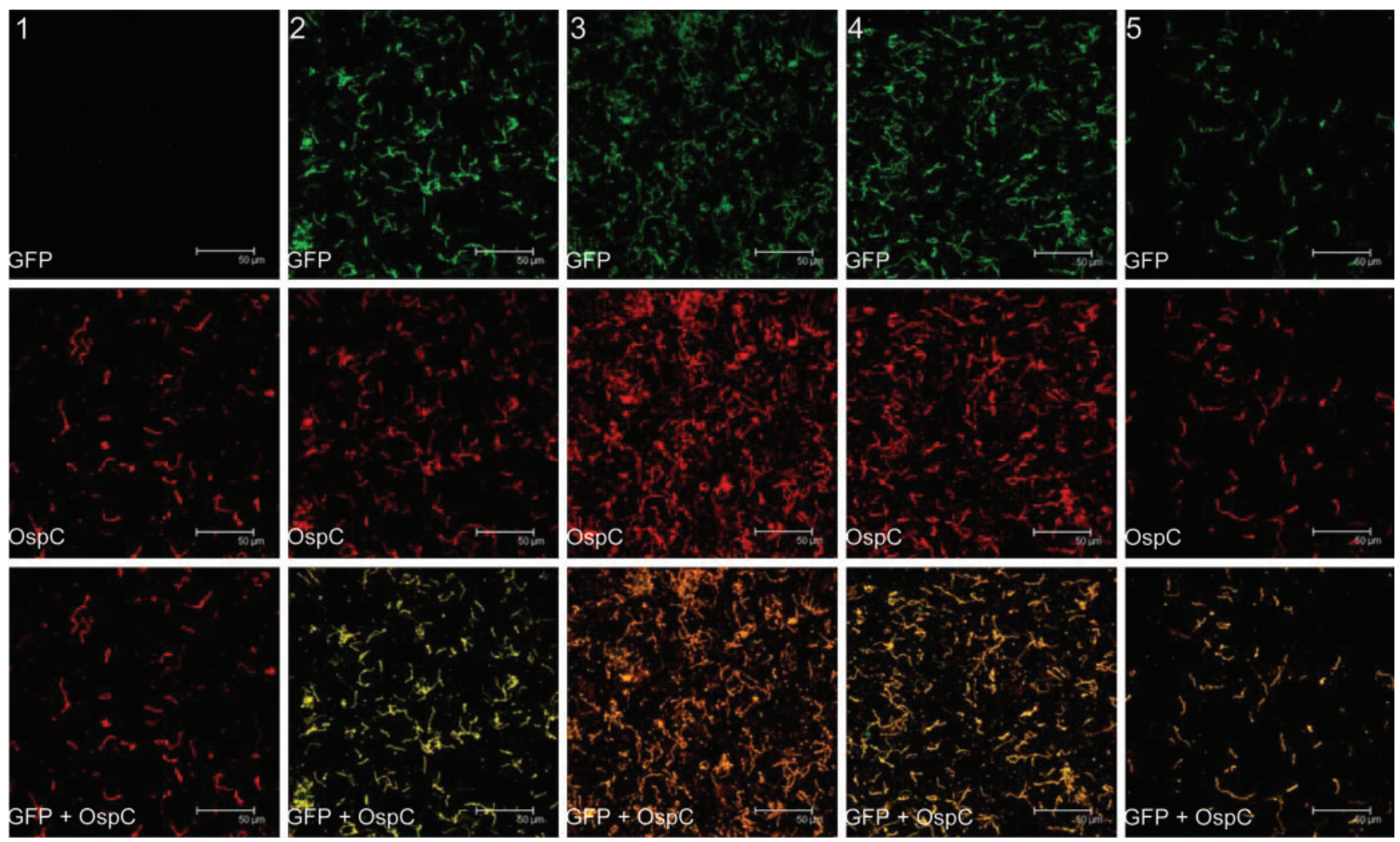

Fig. 6. Co-expression of GFP and OspC in subpopulations of spirochaetes transformed with the various promoter constructs. Slides containing the transformants listed below were stained with an anti-OspC Ab and imaged by confocal microscopy. The GFP and OspC images were merged to assess the expression of these proteins in individual cells. Columns: 1, pBSV2G-gfp; 2, pBSV2G-A64p-gfp; 3, pBSV2G-A64p5'm-gfp; 4, pBSV2G-A64p5'3'm-gfp; 5, pBSV2G-A64pmin-gfp. 
expressed in the same strain with respect to the two culture variables tested, $\mathrm{pH}$ and temperature (Clifton et al., 2006), both of which conditions influence the expression of RpoS (Hübner et al., 2001; Yang et al., 2000, 2003). Therefore, it was crucial to complement the RpoS defect for an unambiguous assessment of its role in the expression of bba64. Complementation of the rpoS mutant restored the expression of bba64 to a level comparable to the wild-type level, thereby definitively establishing a requirement for RpoS for expression. Incidentally, to our knowledge, this is the first report of complementation of a B. burgdorferi rpoS mutant with a chromosomal copy of the wild-type gene. Using this strategy, we were fortunate to restore RpoS to nearly the same level as that observed in the wild-type parental strain. Although chromosomal integration may be challenging as compared to shuttle-vector transformation, it may be the ideal choice in certain cases by circumventing problems associated with plasmid maintenance and/or copy numbers. Finally, the bb0472-bb0473 intergenic chromosomal target should prove useful for targeting other genes for complementation studies.

In addition to RpoS, one other factor may be involved in the expression of bba64. This factor is the putative DNAbinding protein previously demonstrated to specifically bind to the $k 2$ sequence upstream of the gene (Indest \& Philipp, 2000). Surprisingly, however, mutations of the IRS, the most prominent feature within the $k 2$ region, failed to evoke any response vis-à-vis protein expression. Similarly, deletion of the entire upstream sequence beginning with the $k 2$ region also proved to have no effect. Therefore, the expression of GFP in culture appears to utilize just the bba64 basal promoter. It is essential to note that in all cases, the expression of GFP was limited to the same subpopulation of cells that also expressed OspC. Phenotypic heterogeneity of OspC has been previously observed in spirochaetal populations during tick feeding (Schwan et al., 1995; Schwan \& Piesman, 2000) and in culture (Earnhart et al., 2007). Since both OspC and BBA64 require RpoS for expression, it is tantalizing to speculate that in culture only a limited number of spirochaetes express RpoS, or alternatively express higher levels of RpoS, resulting in the observed phenotypic heterogeneity at the population level.

The passivity of the sequence upstream of the bba64 basal promoter in cultured spirochaetes is similar to that reported for the $o s p C$ gene. In the case of $o s p C$, a deletion of the sequence upstream of the promoter, which features an IRS, results in no effect on gene expression in vitro (Yang et al., 2005; Xu et al., 2007). Nonetheless, the IRS, subsequently dubbed the operator, assumes functional significance in vivo, wherein its presence is crucial for the suppression of OspC expression post-infection (Xu et al., 2007). It is very likely that a DNA-binding protein is responsible for this suppression of $o s p C$, although no such protein has yet been reported. In contrast to $o s p C$, a DNAbinding protein specific to bba64 has been shown to be present in cultured spirochaetes (Indest \& Philipp, 2000).
However, the lack of any response from the $k 2$ region suggests that the reported bba64-specific DNA-binding protein is inactive in cultured spirochaetes under the conditions tested. Alternatively, the expression of the bba64-specific DNA-binding protein may be very low or absent in strain B31 5A4NP1, the focus of this study. Notwithstanding, based on its location downstream of the stop codon of $b b a 65$, it is very likely that the $k 2$ region with its IR element functions as a transcription terminator for bba65.

Two reports that are pertinent to the discussion of bba64 regulation must be highlighted. Anguita et al. (2000) noted that the high-passage but infectious strain N40-P75 failed to express bba64 and several other genes now known to be RpoS-regulated in vivo (Fisher et al., 2005), despite a vigorous synthesis of OspC (Anguita et al., 2000) and BBA64 (our unpublished observations) in vitro. The failure to induce gene expression appears to be unrelated to any gross loss of genetic material (Anguita et al., 2000). Therefore, the simplest explanation for these observations is that the in vivo expression of RpoS or some other common factor is defective in this high-passage variant, leading to a broader loss of gene expression. A more recent investigation of gene expression during persistent infection of mice has revealed the down-regulation of bba64 mRNA expression in the ear relative to that in cultured spirochaetes at all time points tested (Gilmore et al., 2007), although importantly, unlike N40-P75, the down-regulation of bba64 mRNA appears in this case to be specific, as the same tissue sample(s) exhibited an upregulation of bba65 and bba66, two other RpoS-dependent genes (Fisher et al., 2005). However, this loss of expression in the ear was countered by the expression of bba64 elsewhere in the body, as these mice continued to harbour anti-BBA64 Abs throughout the course of infection. If these observations hold true, it suggests that bba64 expression in the ear, and perhaps other organs, is repressed. Such repression may well involve the $k 2$ region and the putative bba64-specific DNA-binding protein.

The pattern of expression of bba64 in culture in response to different environmental conditions and during infection of the vertebrate host points to a complex mode of regulation of bba64. Moreover, its expression pattern suggests an important function in establishing and maintaining infection in the vertebrate host. Given this importance, it is crucial to continue to explore the function and regulation of bba64 expression and assess its role in virulence and pathogenesis. Finally, understanding the function and regulation of this molecule may also shed light on the orchestration of regulation of the other members of the gbb54 gene family and their contribution to the overall molecular strategies of this pathogen.

\section{ACKNOWLEDGEMENTS}

We thank Dr Patricia Rosa, Rocky Mountain Laboratories, Hamilton, $\mathrm{MT}$, for the gifts of the pBSV2G vector, Dr Steve Norris and Dr 
Hiroki Kawabata, Texas Medical School, Houston, TX, for the bbe02 knockout strain B31 5A4NP1, and Dr Frank Gherardini, Rocky Mountain Laboratories, for the B31 A3 and B31 A3rpoS mutant strains. We thank Xavier Alvarez for helpful suggestions relating to confocal microscopy. We also thank Mario Philipp for helpful comments. This work was funded by grant AI 49293 from the National Institute of Allergy and Infectious Diseases, National Institutes of Health.

Portions of this work were presented at the American Society of Microbiology meeting in Atlanta, June 2005, and at the Gordon Conference on the Biology of Spirochaetes, Il Ciocco, Italy, April 2006.

\section{REFERENCES}

Anguita, J., Samanta, S., Revilla, B., Suk, K., Das, S., Barthold, S. W. \& Fikrig, E. (2000). Borrelia burgdorferi gene expression in vivo and spirochete pathogenicity. Infect Immun 68, 1222-1230.

Barbour, A. G. \& Garon, C. F. (1987). Linear plasmids of the bacterium Borrelia burgdorferi have covalently closed ends. Science 237, 409-411.

Brooks, C. S., Hefty, P., Scott, J., Sarah, E. \& Akins, D. R. (2003). Global analysis of Borrelia burgdorferi genes regulated by mammalian host-specific signals. Infect Immun 71, 3371-3383.

Brooks, C. S., Vuppula, S. R., Jett, A. M., Alitalo, A., Meri, S. \& Akins, D. R. (2005). Complement regulator-acquiring surface protein 1 imparts resistance to human serum in Borrelia burgdorferi. J Immunol 175, 3299-3308.

Brooks, C. S., Vuppala, S. R., Jett, A. M. \& Akins, D. R. (2006). Identification of Borrelia burgdorferi outer surface proteins. Infect Immun 74, 296-304.

Caimano, M. J., Eggers, C. H., Gonzales, C. A. \& Radolf, J. D. (2005). Alternate sigma factor RpoS is required for the in vivo-specific repression of Borrelia burgdorferi plasmid lp54-borne ospA and lp6.6 genes. J Bacteriol 187, 7845-7852.

Carroll, J. A., Cordova, R. M. \& Garon, C. F. (2000). Identification of $11 \mathrm{pH}$-regulated genes in Borrelia burgdorferi localizing to linear plasmids. Infect Immun 68, 6677-6684.

Carroll, J. A., Stewart, P. E., Rosa, P., Elias, A. F. \& Garon, C. F. (2003). An enhanced GFP reporter system to monitor gene expression in Borrelia burgdorferi. Microbiology 149, 1819-1828.

Casjens, S., Palmer, N., van Vugt, R., Huang, W. M., Stevenson, B., Rosa, P., Lathigra, R., Sutton, G., Peterson, J. \& other authors (2000). A bacterial genome in flux: the twelve linear and nine circular extrachromosomal DNAs in an infectious isolate of the Lyme disease spirochaete Borrelia burgdorferi. Mol Microbiol 35, 490-516.

Clifton, D. R., Nolder, C. L., Hughes, J. L., Nowalk, A. J. \& Carroll, J. A. (2006). Regulation and expression of bba66 encoding an immunogenic infection-associated lipoprotein in Borrelia burgdorferi. Mol Microbiol 61, 243-258.

Earnhart, C. G., Buckles, E. L. \& Marconi, R. T. (2007). Development of an OspC-based tetravalent, recombinant, chimeric vaccinogen that elicits bactericidal antibody against diverse Lyme disease spirochete strains. Vaccine 25, 466-480.

Elias, A. F., Stewart, P. E., Grimm, D., Caimano, M. J., Eggers, C. H., Tilly, K., Bono, J. L., Akins, D. R., Radolf, J. D. \& other authors (2002). Clonal polymorphism of Borrelia burgdorferi strain B31 MI: implications for mutagenesis in an infectious strain background. Infect Immun 70, 2139-2150.

Elias, A. F., Bono, J. L., Kupko, J. J., 3rd, Stewart, P. E., Krum, J. G. \& Rosa, P. A. (2003). New antibiotic resistance cassettes suitable for genetic studies in Borrelia burgdorferi. J Mol Microbiol Biotechnol 6, $29-40$.
Fisher, M. A., Grimm, D., Henion, A. K., Elias, A. F., Stewart, P. E., Rosa, P. A. \& Gherardini, F. C. (2005). Borrelia burgdorferi $\sigma^{54}$ is required for mammalian infection and vector transmission but not for tick colonization. Proc Natl Acad Sci U S A 102, 5162-5167.

Frank, K. L., Bundle, S. F., Kresge, M. E., Eggers, C. H. \& Samuels, D. S. (2003). aadA confers streptomycin resistance in Borrelia burgdorferi. J Bacteriol 185, 6723-6727.

Fraser, C. M., Casjens, S., Huang, W. M., Sutton, G. G., Clayton, R., Lathigra, R., White, O., Ketchum, K. A., Dodson, R. \& other authors (1997). Genomic sequence of a Lyme disease spirochaete, Borrelia burgdorferi. Nature 390, 580-586.

Gilmore, R. D., Jr, Kappel, K. J., Jr \& Johnson, B. J. (1997). Molecular characterization of a 35-kilodalton protein of Borrelia burgdorferi, an antigen of diagnostic importance in early Lyme disease. J Clin Microbiol 35, 86-91.

Gilmore, R. D., Jr, Mbow, M. L. \& Stevenson, B. (2001). Analysis of Borrelia burgdorferi gene expression during life cycle phases of the tick vector Ixodes scapularis. Microbes Infect 3, 799-808.

Gilmore, R. D., Jr, Howison, R. R., Schmit, V. L., Nowalk, A. J., Clifton, D. R., Nolder, C., Hughes, J. L. \& Carroll, J. A. (2007). Temporal expression analysis of the Borrelia burgdorferi paralogous gene family 54 genes BBA64, BBA65, and BBA66 during persistent infection in mice. Infect Immun 75, 2753-2764.

Grimm, D., Eggers, C. H., Caimano, M. J., Tilly, K., Stewart, P. E., Elias, A. F., Radolf, J. D. \& Rosa, P. A. (2004). Experimental assessment of the roles of linear plasmids lp25 and lp28-1 of Borrelia burgdorferi throughout the infectious cycle. Infect Immun 72, 5938-5946.

Hagman, K. E., Lahdenne, P., Popova, T. G., Porcella, S. F., Akins, D. R., Radolf, J. D. \& Norgard, M. V. (1998). Decorin-binding protein is encoded within a two-gene operon and is protective in the murine model of Lyme borreliosis. Infect Immun 66, 2674-2683.

Hübner, A., Yang, X., Nolen, D. M., Popova, T. G., Cabello, F. C. \& Norgard, M. V. (2001). Expression of Borrelia burgdorferi OspC and DbpA is controlled by a RpoN-RpoS regulatory pathway. Proc Natl Acad Sci U S A 98, 12724-12729.

Indest, K. J. \& Philipp, M. T. (2000). DNA-binding proteins possibly involved in regulation of the post-logarithmic-phase expression of lipoprotein P35 in Borrelia burgdorferi. J Bacteriol 182, 522-525.

Indest, K. J., Ramamoorthy, R., Sole, M., Gilmore, R. D., Johnson, B. J. \& Philipp, M. T. (1997). Cell-density-dependent expression of Borrelia burgdorferi lipoproteins in vitro. Infect Immun 65, 1165-1171.

Kawabata, H., Norris, S. J. \& Watanabe, H. (2004). BBE02 disruption mutants of Borrelia burgdorferi B31 have a highly transformable, infectious phenotype. Infect Immun 72, 7147-7154.

Kraiczy, P., Hellwage, J., Skerka, C., Becker, H., Kirschfink, M., Simon, M. M., Brade, V., Zipfel, P. F. \& Wallich, R. (2004). Complement resistance of Borrelia burgdorferi correlates with the expression of BbCRASP-1, a novel linear plasmid-encoded surface protein that interacts with human factor $\mathrm{H}$ and FHL-1 and is unrelated to Erp proteins. J Biol Chem 279, 2421-2429.

Labandeira-Rey, M. \& Skare, J. T. (2001). Decreased infectivity in Borrelia burgdorferi strain B31 is associated with loss of linear plasmid lp25 or lp28-1. Infect Immun 69, 446-455.

Liang, F. T., Nelson, F. K. \& Fikrig, E. (2002). DNA microarray assessment of putative Borrelia burgdorferi lipoprotein genes. Infect Immun 70, 3300-3303.

Mbow, M. L., Gilmore, R. D., Jr \& Titus, R. G. (1999). An OspC-specific monoclonal antibody passively protects mice from tick-transmitted infection by Borrelia burgdorferi B31. Infect Immun 67, 5470-5472.

McDowell, J. V., Harlin, M. E., Rogers, E. A. \& Marconi, R. T. (2005). Putative coiled-coil structural elements of the BBA68 protein of Lyme 
disease spirochetes are required for formation of its factor $\mathrm{H}$ binding site. J Bacteriol 187, 1317-1323.

McDowell, J. V., Hovis, K. M., Zhang, H., Tran, E., Lankford, J. \& Marconi, R. T. (2006). Evidence that the BBA68 protein (BbCRASP-1) of the Lyme disease spirochetes does not contribute to factor $\mathrm{H}$ mediated immune evasion in humans and other animals. Infect Immun 74, 3030-3034.

Nowalk, A. J., Gilmore, R. D., Jr \& Carroll, J. A. (2006). Serologic proteome analysis of Borrelia burgdorferi membrane-associated proteins. Infect Immun 74, 3864-3873.

Ojaimi, C., Brooks, C., Casjens, S., Rosa, P., Elias, A., Barour, A., Jasinskas, A., Benach, J., Katona, L. \& other authors (2003). Profiling of temperature-induced changes in Borrelia burgdorferi gene expression by using whole genome arrays. Infect Immun 71, 1689-1705.

Purser, J. E. \& Norris, S. J. (2000). Correlation between plasmid content and infectivity in Borrelia burgdorferi. Proc Natl Acad Sci U S A 97, 13865-13870.

Ramamoorthy, R. \& Philipp, M. T. (1998). Differential expression of Borrelia burgdorferi proteins during growth in vitro. Infect Immun 66, 5119-5124.

Ramamoorthy, R. \& Scholl-Meeker, D. (2001). Borrelia burgdorferi proteins whose expression is similarly affected by culture temperature and $\mathrm{pH}$. Infect Immun 69, 2739-2742.

Ramamoorthy, R., Povinelli, L. \& Philipp, M. T. (1996). Molecular characterization, genomic arrangement, and expression of $b m p D$, a new member of the $b m p$ class of genes encoding membrane proteins of Borrelia burgdorferi. Infect Immun 64, 1259-1264.

Ramamoorthy, R., McClain, N. A., Gautam, A. \& Scholl-Meeker, D. (2005). Expression of the $b m p B$ gene of Borrelia burgdorferi is modulated by two distinct transcription termination events. J Bacteriol 187, 2592-2600.

Revel, A. T., Talaat, A. M. \& Norgard, M. V. (2002). DNA microarray analysis of differential gene expression in Borrelia burgdorferi, the Lyme disease spirochaete. Proc Natl Acad Sci U S A 99, 1562-1567.

Samuels, D. S. (1995). Electrotransformation of the spirochete Borrelia burgdorferi. Methods Mol Biol 47, 253-259.

Schwan, T. G. \& Piesman, J. (2000). Temporal changes in outer surface proteins $\mathrm{A}$ and $\mathrm{C}$ of the Lyme disease-associated spirochete Borrelia burgdorferi, during the chain of infection in ticks and mice. J Clin Microbiol 38, 382-388.
Schwan, T. G., Piesman, J., Golde, W. T., Dolan, M. C. \& Rosa, P. A. (1995). Induction of an outer surface protein on Borrelia burgdorferi during tick feeding. Proc Natl Acad Sci U S A 92, 2909-2913.

Sohaskey, C. D., Zückert, W. R. \& Barbour, A. G. (1999). The extended promoters for two outer membrane lipoprotein genes of Borrelia spp. uniquely include a T-rich region. Mol Microbiol 33, 41-51.

Sung, S. Y., McDowell, J. V., Carlyon, J. A. \& Marconi, R. T. (2000). Mutation and recombination in the upstream homology box-flanked ospE-related genes of the Lyme disease spirochetes result in the development of new antigenic variants during infection. Infect Immun 68, 1319-1327.

Tokarz, R., Anderton, J. M., Katona, L. I. \& Benach, J. L. (2004). Combined effects of blood and temperature shift on Borrelia burgdorferi gene expression as determined by whole genome DNA array. Infect Immun 72, 5419-5432.

Wallich, R., Pattathu, J., Kitiratschky, V., Brenner, C., Zipfel, P. F., Brade, V., Simon, M. M. \& Kraiczy, P. (2005). Identification and functional characterization of complement regulator-acquiring surface protein 1 of the Lyme disease spirochetes Borrelia afzelii and Borrelia garinii. Infect Immun 73, 2351-2359.

Xu, Q., McShan, K. \& Liang, F. T. (2007). Identification of an $\operatorname{sp} p C$ operator critical for immune evasion of Borrelia burgdorferi. Mol Microbiol 64, 220-231.

Yang, X. F., Goldberg, M. S., Popova, T. G., Schoeler, G. B., Wikel, S. K., Hagman, K. E. \& Norgard, M. V. (2000). Interdependence of environmental factors influencing reciprocal patterns of gene expression in virulent Borrelia burgdorferi. Mol Microbiol 37, 1470-1479.

Yang, X. F., Alani, S. M. \& Norgard, M. V. (2003). The response regulator Rrp2 is essential for the expression of major membrane lipoproteins in Borrelia burgdorferi. Proc Natl Acad Sci U S A 100, 11001-11006.

Yang, X. F., Pal, U., Alani, S. M., Fikrig, E. \& Norgard, M. V. (2004). Essential role for OspA/B in the life cycle of the Lyme disease spirochaete. J Exp Med 199, 641-648.

Yang, X. F., Lybecker, M. C., Pal, U., Alani, S. M., Blevins, J., Revel, A. T., Samuels, D. S. \& Norgard, M. V. (2005). Analysis of the ospC regulatory element controlled by the RpoN-RpoS regulatory pathway in Borrelia burgdorferi. J Bacteriol 187, 4822-4829.

Edited by: R. J. Lamont 